Benjamin Hofner, Torsten Hothorn and Thomas Kneib

\title{
Variable Selection and Model Choice in Structured Survival Models
}

Technical Report Number 043, 2008 Department of Statistics

University of Munich

http://www.stat.uni-muenchen.de 


\title{
Variable Selection and Model Choice in Structured Survival Models
}

\author{
Benjamin Hofner* Torsten Hothorn ${ }^{\dagger} \quad$ Thomas Kneib ${ }^{\dagger}$
}

\begin{abstract}
In many situations, medical applications ask for flexible survival models that allow to extend the classical Cox-model via the inclusion of time-varying and nonparametric effects. These structured survival models are very flexible but additional difficulties arise when model choice and variable selection is desired. In particular, it has to be decided which covariates should be assigned timevarying effects or whether parametric modeling is sufficient for a given covariate. Component-wise boosting provides a means of likelihood-based model fitting that enables simultaneous variable selection and model choice. We introduce a component-wise likelihood-based boosting algorithm for survival data that permits the inclusion of both parametric and nonparametric time-varying effects as well as nonparametric effects of continuous covariates utilizing penalized splines as the main modeling technique. Its properties and performance are investigated in simulation studies. The new modeling approach is used to build a flexible survival model for intensive care patients suffering from severe sepsis. A software implementation is available to the interested reader.
\end{abstract}

Key words: likelihood-based boosting, hazard regression, model choice, P-splines, smooth effects, time-varying effects

\section{Introduction}

Classical survival models had a break-through with the well-known, omnipresent Cox model [1], where the hazard rate is described in terms of a baseline hazard and multiplicative covariate effects. Modeling more complex survival regression relationships requires a more flexible model structure and in particular calls for smooth, nonlinear and time-varying effects. Here, we focus on building a model for the survival time of intensive care patients suffering from severe sepsis. Previously published findings, based on survival models estimated for 462 patients which were enrolled in a study initiated at the university hospital "Klinikum Großhadern" of the Ludwig-Maximilians-Universität München, suggest that out of potentially 20 covariates, 14 covariates have an impact on the survival time or were set as mandatory covariates [2]. The model was derived based on the recently developed two-stage stepwise procedure of Hofner et al. [3]. To illustrate the type of models we will consider in the following, we chose three exemplary covariates of the 14 covariates that were identified to have an impact on survival times: "age" $\left(x_{1}\right)$ was selected as linear term, "Apache II score" $\left(x_{2}\right.$, a measure for the severity of disease determined within the first 24 hours of admission) had a smooth effect and "fungal infection" $\left(x_{3}\right)$ was modeled with time-varying effect. In other words, the following structured, flexible survival model for the hazard rate has to be fitted

$$
\lambda(t \mid \boldsymbol{x})=\lambda_{0}(t) \exp \left\{f_{\text {linear }}\left(x_{1}\right)+f_{\text {smooth }}\left(x_{2}\right)+f_{\text {smooth }}(t) \cdot x_{3}\right\},
$$

where $\boldsymbol{x}=\left(x_{1}, x_{2}, x_{3}\right)^{\top}$ is the vector of covariates, $\lambda_{0}(t)$ represents the baseline hazard, $f_{\text {linear }}\left(x_{1}\right)$ is a linear function of "age" $\left(x_{1}\right), f_{\text {smooth }}\left(x_{2}\right)$ is a smooth function of "Apache II score" $\left(x_{2}\right)$, and

\footnotetext{
${ }^{*}$ Institut für Medizininformatik, Biometrie und Epidemiologie, Friedrich-Alexander-Universität Erlangen-Nürnberg, Waldstraße 6, 91054 Erlangen, Germany; Email: benjamin.hofner@imbe.med.uni-erlangen.de

${ }^{\dagger}$ Institut für Statistik; Ludwig-Maximilians-Universität München
} 
$f_{\text {smooth }}(t)$ is again a function of time $t$, which represents the time-varying effect of "fungal infection" $\left(x_{3}\right)$. Once one is sure about the principle structure of such a complex model, various approaches to model fitting can be applied. Basics of Cox-type additive models can be found, e.g., in Zucker and Karr [4] or Fahrmeir et al. [5]. Hastie and Tibshirani [6] introduced models with varying-coefficients and also considered time-varying effects in Cox models as a specific example. Time-varying effects can be expressed as the product of a P-spline and the covariate (e.g., [7]). This can be seen in $f_{\text {smooth }}(t) \cdot x_{3}$, where $f_{\text {smooth }}(t)$ is modeled using $\mathrm{P}$-splines. The smooth function $f_{\text {smooth }}\left(x_{2}\right)$ can also be modeled using P-splines.

However, the crucial point is not simply fitting a model similar to (1) but to derive a model structure describing the response in terms of only influential covariates at an appropriate complexity. In model (1), one might ask why a smooth term for $x_{2}$ is required whereas a simple linear term seems to be sufficient to capture the impact of $x_{1}$. In principle, we have to decide what the appropriate complexity (linear effect, smooth effect or time-varying effect) for each variable is. This decision has to be based on data and thus we are faced with a model choice problem. Even more crucial is the necessity to distinguish between influential and non-influential covariates. A covariate is influential if it is related to the response in any of the given modeling possibilities and non-influential if it is independent of the response. Thus, in addition to the model choice problem, we are faced with a variable selection problem and need not only to select variables but also to determine the appropriate structure for the covariate at the same time.

In classical structured Cox-type additive models it is hard to deal with both the model choice and variable selection problem. It is often not clear if a covariate should enter the model as linear term, smooth term or as time-varying effect, or even not enter the model at all. One approach to address this problem are multivariable fractional polynomials [8]. The basic idea is to start with the simplest model and to apply an iterative variable inclusion procedure based on a series of tests for inclusion of variables and for determining the complexity of the functional form. A two-stage stepwise variable selection and model choice algorithm for structured Cox-type additive models based on information criteria (as e.g., AIC or BIC) is suggested by Hofner et al. [3] and was applied to model the severe sepsis data. Other approaches that are based on hypothesis tests may be applied as well [9]. These and similar multi-step procedures perform a series of locally optimal fit and selection procedures, however, the quality of the global model fit can only be investigated empirically.

To overcome these conceptual and associated practical problems, we propose a one-step model fitting approach with intrinsic variable selection and model choice within the framework of empirical risk minimization. Our suggestion is to fit structured survival models with potentially many covariates by minimizing the respective negative (log)-likelihood based on boosting techniques. More specifically, a component-wise boosting approach is applied which allows for estimation of structured models and has been shown to lead to final models containing influential variables at appropriate complexity, for example in [10]. Over the last twelve years, an extensive amount of literature has been devoted to boosting techniques and we therefore refer the reader to Bühlmann and Hothorn [11] for an overview. In a context not unlike the one dealt with in this paper, Kneib et al. [12] studied boosting techniques for estimation, variable selection and model choice for spatially structured exponential family regression models.

The rest of the paper is organized as follows: Structured survival models and penalized likelihood estimation schemes that will be utilized as building blocks in the boosting algorithm are introduced in Section 2. Section 3 a component-wise, likelihood-based boosting method that allows for simultaneous model choice and estimation. A simulation study to investigate the characteristics of the algorithm is given in Section 4. The application of the algorithm to the severe sepsis data, as briefly introduced above, is also presented in this section, along with an empirical comparison with the two-stage stepwise selection procedure by Hofner et al. [3]. Section 5 contains a discussion of the proposed method and presents an outlook. 


\section{Structured Survival Models}

To overcome the restrictions of Cox models, as discussed above, we allow the inclusion of both, timevarying and smooth effects. For methodological considerations take a generic, structured survival model

$$
\lambda\left(t \mid \boldsymbol{x}_{i}\right)=\exp \left(\eta_{i}\left(\boldsymbol{x}_{i}\right)\right), i=1, \ldots, n,
$$

with an additive predictor of the form

$$
\eta_{i}\left(\boldsymbol{x}_{i}\right)=\beta_{0}+\sum_{j=1}^{J} f_{j}\left(\boldsymbol{x}_{\boldsymbol{i}}\right),
$$

where the functions $f_{j}\left(\boldsymbol{x}_{i}\right)$ are a generic representation of different types of covariate effects. To make the model formulation (3) more concrete, consider the following examples of functions $f_{j}(\boldsymbol{x})$ : The functions can represent linear effects $f_{j}(\boldsymbol{x})=f_{\text {linear }}(\tilde{x})=\tilde{x} \beta$, where $\tilde{x}$ is a univariate element of $\boldsymbol{x}$, smooth effects $f_{j}(\boldsymbol{x})=f_{\text {smooth }}(\tilde{x})$, where $\tilde{x}$ is a univariate, continuous covariate from $\boldsymbol{x}$ and $f_{\text {smooth }}$ is a smooth function, and time-varying effects $f_{j}(\boldsymbol{x})=f_{\text {smooth }}(t) \cdot \tilde{x}$, where the vector $(\tilde{x}, t)$ is included in $\boldsymbol{x}$. The covariate $\tilde{x}$ can be either continuous or categorical, $t$ represents the observed survival time and $f_{\text {smooth }}$ is again a smooth function. The log-baseline hazard can be specified in the additive predictor (3) as a special form of the generic functions, i.e., $f_{j}=f_{\text {smooth }}(t)=\log \left(\lambda_{0}(t)\right)$. Thus, there is no need to additionally specify the classical baseline hazard $\lambda_{0}(t)$ in the model (2). Furthermore, the full likelihood is available and thus can be used for inference.

The smooth functions $f_{\text {smooth }}$, of $\tilde{x}$ as well as of time $t$, can be modeled, for example, by applying fractional polynomials $[13,14]$. Perperoglou et al. [15] propose the reduced-rank regression model to model time-varying effects based on B-splines. Both, flexible and time-varying effects can be modeled by classical smoothers as regression splines, smoothing splines [7] or P-splines. The latter where introduced by Eilers and Marx [16] based on B-splines [17], where smooth functions are modeled using a parametric analogon

$$
f_{\text {smooth }}(\tilde{x})=\sum_{m=1}^{M} \beta_{m} B_{m}(\tilde{x}) .
$$

The B-spline basis functions $B_{m}(\tilde{x})$ are defined over an equidistant grid of $M$ knots. The coefficients $\beta_{m}$ become part of the vector of unknown parameters we want to estimate. An additional penalty for higher order differences of coefficients $\beta_{m}$ for adjacent knots is used to achieve smooth estimates. For the $j$-th function $f_{j}(\tilde{\boldsymbol{x}})=f_{\text {smooth }}(\tilde{\boldsymbol{x}})$, we get the parameter vector $\boldsymbol{\beta}_{\mathrm{pen}, j}=\left(\beta_{1, j}, \ldots, \beta_{M, j}\right)^{\top}$ and the design matrix $\boldsymbol{X}_{\mathrm{pen}, j}=\left(B_{1, j}(\tilde{\boldsymbol{x}}), \ldots, B_{M, j}(\tilde{\boldsymbol{x}})\right)$, where $\tilde{\boldsymbol{x}}$ is the covariate vector corresponding to the $j$-th generic function. Numerically, P-splines are very stable and the computational effort is reduced compared to, e.g., smoothing splines. In the survival context, P-splines are frequently used to model smooth functions [18]. Schmid and Hothorn [19] showed that P-splines can also be successfully used in the boosting context. They reason that the computational effort is heavily decreased and the predictive performance is only marginally effected when using P-splines instead of smoothing splines. Following this argumentation we use $\mathrm{P}$-splines as base-learners for flexible model terms in the remainder of this paper.

To estimate the structured, flexible survival model, we need to derive the penalized components for likelihood estimation in this context. The likelihood will then be maximized by applying a componentwise boosting approach. Let $t_{i}$ denote the observed survival time of the $i$-th observation $(i=1, \ldots, n)$ and $\delta_{i}$ be the corresponding non-censoring indicator. Under non-informative censoring, the (penalized) 
log-likelihood can be expressed as

$$
\begin{aligned}
l_{\mathrm{pen}}(\boldsymbol{\beta}) & =\sum_{i=1}^{n}\left(\delta_{i} \eta_{i}-\int_{0}^{t_{i}} \lambda_{i}(\tilde{t}) d \tilde{t}\right)-\sum_{j=1}^{J} \frac{\kappa_{j}}{2} \boldsymbol{\beta}_{\mathrm{pen}, j}^{\top} \boldsymbol{K}_{j} \boldsymbol{\beta}_{\mathrm{pen}, j} \\
& =\left[\boldsymbol{\delta}^{\top} \boldsymbol{\eta}-\mathbb{1}^{\top} \boldsymbol{\Lambda}\right]-\frac{1}{2} \boldsymbol{\beta}^{\top} \boldsymbol{K} \boldsymbol{\beta} \\
& =l(\boldsymbol{\beta})-\frac{1}{2} \boldsymbol{\beta}^{\top} \boldsymbol{K} \boldsymbol{\beta},
\end{aligned}
$$

where $\boldsymbol{\beta}^{\top}=\left(\boldsymbol{\beta}_{\text {pen, } 1}^{\top}, \ldots, \boldsymbol{\beta}_{\text {pen }, J}^{\top}, \boldsymbol{\gamma}^{\top}\right)$ is the parameter vector, $\boldsymbol{\Lambda}=\left(\Lambda_{1}\left(t_{1}\right), \ldots, \Lambda_{n}\left(t_{n}\right)\right)^{\top}$ is the vector of the cumulative hazard rates $\Lambda_{i}\left(t_{i}\right)=\int_{0}^{t_{i}} \lambda_{i}(\tilde{t}) d \tilde{t}, l(\boldsymbol{\beta})$ denotes the unpenalized log-likelihood and $\boldsymbol{K}=\operatorname{diag}\left(\kappa_{1} \boldsymbol{K}_{1}, \ldots, \kappa_{J} \boldsymbol{K}_{J}, \mathbf{0}\right)$ is a block diagonal matrix representing the penalization. $\boldsymbol{K}_{j}$ is a difference matrix (classically of order two) for the $j$-th component and $\kappa_{j}$ is the corresponding smoothing parameter. The latter determines the smoothness of the resulting function estimate, where bigger values of $\kappa_{j}$ correspond to smoother functions and $\kappa_{j}=0$ corresponds to an unpenalized estimation of the $j$-th term. Note that the smoothing parameters $\kappa_{j}, j=1, \ldots, J$ are part of $\boldsymbol{K}$ and linear effects remain unpenalized in the model, only coefficients corresponding to smooth terms are penalized.

The score vector is given by the first derivative of the (penalized) $\log$-likelihood $l_{\text {pen }}(\boldsymbol{\beta})$

$$
\begin{aligned}
\boldsymbol{s}_{\mathrm{pen}}(\boldsymbol{\beta}) & =\frac{\partial}{\partial \boldsymbol{\beta}} l_{\text {pen }}(\boldsymbol{\beta}) \\
& =\left[\boldsymbol{\delta}^{\top} \boldsymbol{X}-\sum_{i=1}^{n} \int_{0}^{t_{i}} \boldsymbol{x}_{i}(\tilde{t}) \lambda_{i}(\tilde{t}) d \tilde{t}\right]-\boldsymbol{K} \boldsymbol{\beta} \\
& =\boldsymbol{s}(\boldsymbol{\beta})-\boldsymbol{K} \boldsymbol{\beta},
\end{aligned}
$$

where the unpenalized score function is denoted by $\boldsymbol{s}(\boldsymbol{\beta})$. The notation $\boldsymbol{x}_{i}(\tilde{t})$ depicts that $\boldsymbol{x}_{i}$ may contain time-depending covariates. This can, for example, be the case when time-varying effects are used, as these are expressed as artificial time-dependent covariates. The penalized, observed Fisher matrix is then calculated as the negative second derivative of the penalized log-likelihood:

$$
\begin{aligned}
\boldsymbol{F}_{\text {pen }}(\boldsymbol{\beta}) & =-\frac{\partial^{2}}{\partial \boldsymbol{\beta} \partial \boldsymbol{\beta}^{\top}} l_{\text {pen }}(\boldsymbol{\beta}) \\
& =\left[\sum_{i=1}^{n} \int_{0}^{t_{i}} \boldsymbol{x}_{i}(\tilde{t}) \boldsymbol{x}_{i}^{\top}(\tilde{t}) \lambda_{i}(\tilde{t}) d \tilde{t}\right]+\boldsymbol{K} \\
& =\boldsymbol{F}(\boldsymbol{\beta})+\boldsymbol{K} .
\end{aligned}
$$

The Fisher matrix that results from unpenalized estimation is given as $\boldsymbol{F}(\boldsymbol{\beta})$. With these formulations at hand one can estimate the parameters using Fisher scoring or any other numerical optimization method.

In most cases it is better to define the smoothness of a function using the effective degrees of freedom df than to set the smoothing parameters $\kappa_{j}, j=1, \ldots, J$ itself. This makes functions comparable w.r.t. their flexibility (i.e. smoothness) and is more intuitive. Gray [7] derived the degrees of freedom in flexible survival models with penalized splines as

$$
\mathrm{df}:=\operatorname{trace}\left[\boldsymbol{F} \cdot \boldsymbol{F}_{\mathrm{pen}}^{-1}\right] .
$$

Note that the degrees of freedom depend on the smoothing parameters $\kappa_{j}, j=1, \ldots, J$, the parameters $\boldsymbol{\beta}$ and on the observed survival times $t_{i}, i=1, \ldots, n$. 


\section{Boosting in Survival Models with Time-Varying Effects}

In this section, we device an estimation procedure for Cox-type models with additive structure and possibly time-varying effects. Variable selection and model choice play another major role in this setting. To combine all tasks, component-wise boosting methods are applied in the following.

\subsection{Basic Considerations}

Estimation of models can be done with respect to many different criteria. In the boosting context, minimization of a loss function based on the negative gradient (functional gradient descent [FGD] boosting) or direct maximization of a likelihood-based criterion (likelihood-based boosting) is usually applied. We restrict to the latter case and base the estimation on the full log-likelihood (in contrast to the usually applied partial log-likelihood). Likelihood-based boosting directly aims to maximize the log-likelihood and thus is to be understood as a special algorithm for the maximization of the likelihood. Boosting is based on base-learners, i.e., functions that lead to (typically) small improvements of the estimation in each boosting iteration. Thus, we are slowly approaching a solution. For more details on base-learners in general and on how to choose them we refer to Bühlmann and Hothorn [11]. In the following, we will consider linear and P-spline base-learners. For the latter base-learners, the optimization criterion is altered from the unpenalized to the penalized log-likelihood as given in (5).

Variable Selection To incorporate variable selection, component-wise boosting [10] is employed. For each covariate a separate base-learner is specified and only the best fitting base-learner (w.r.t. some criterion) is updated in each iteration. Hence, classically we do not incorporate each base-learner in the model before we reach the "optimal" boosting iteration, which means that variable selection is performed.

Model Choice To incorporate model choice in the (component-wise) boosting framework we add separate base-learners for each modeling possibility. A variable can then be added in any of the modeling possibilities, which corresponds to model choice. Furthermore, a variable is considered to be selected if any of the modeling possibilities is chosen. Thus, we have a variable selection and model choice approach based on component-wise boosting.

From the generic, flexible survival model $(2,3)$ we see that a covariate $\tilde{x}_{i}$ can enter the model in up to three different ways. The effect can be either linear, smooth (in the case of a continuous covariate $\tilde{x}_{i}$ ) or time-varying. Hence, the question arises, how each variable should enter the model. One solution is, to specify a separate base-learner for each suitable modeling possibility. Component-wise boosting then chooses between covariates and modeling possibilities at the same time, if the boosting algorithm is stopped after an appropriate number of iterations. Linear effects enter the model as linear base-learners, smooth effects can be added using P-spline base-learners and time-varying effects are derived as a base-learner for the interaction between a P-spline of time and the covariate $\tilde{x}_{i}$.

To make the different base-learners comparable in terms of complexity, one could try to define equal degrees of freedom for each term. Increasing the smoothing parameter $\kappa$ leads to decreasing degrees of freedom. However, Eilers and Marx [16] showed that a polynomial of order $d-1$ remains unpenalized by a $d$-th order difference penalty if the degree of the B-spline basis is larger or equal than $d-1$. Thus, we cannot make the degrees of freedom arbitrary small. As classically we are using B-splines of degree 3 or higher, the degrees of freedom for difference penalties of order 2 or higher remain always greater than one. Hence, making such smooth base-learners comparable with a single linear base-learner seems impossible.

Kneib et al. [12] propose a modified parameterization of the P-splines. Therefore, with a continuous covariate $x$, the smooth function $f_{\text {smooth }}(x)$ is split into a parametric part consisting of the unpenalized 
polynomial of order $d-1$ and the nonparametric deviation from this polynomial $f_{\text {centered }}(x)$ :

$$
f_{\text {smooth }}(x)=\underbrace{\beta_{0}+\beta_{1} x+\ldots+\beta_{d-1} x^{d-1}}_{\text {unpenalized, parametric part }}+\underbrace{f_{\text {centered }}(x)}_{\text {nonparametric deviation from polynomial }}
$$

For the parametric part, separate linear base-learners are added for each term. The deviation from the polynomial $f_{\text {centered }}$ can be included as smooth effect with exactly one degree of freedom. Thus, we have the possibility to check, if $x$ has any influence at all (i.e., none of the base-learners depending on $x$ are selected). If $x$ is influential, we have the additional possibility to check whether we need a nonparametric part to describe the influence.

Varying coefficient terms [6], as time-varying effects can be reparameterized in the same manner, i.e.,

$$
f_{\text {smooth }}(t) \cdot x=\underbrace{\beta_{0} \cdot x+\beta_{1} t \cdot x+\ldots+\beta_{d-1} t^{d-1} \cdot x}_{\text {unpenalized, parametric part }}+\underbrace{f_{\text {centered }}(t) \cdot x}_{\text {nonparametric deviation from polynomial }},
$$

where $t$ is the time and $x$ is an arbitrary covariate.

Technically, this model decomposition is achieved by decomposing the vector of regression coefficients $\boldsymbol{\beta}$ into $\left(\widetilde{\boldsymbol{\beta}}_{\text {unpen }}, \widetilde{\boldsymbol{\beta}}_{\text {pen }}\right)^{\prime}$, i.e., into an unpenalized and a penalized part. This can be achieved based on a spectral decomposition of the penalty matrix. Details in the context of geoadditive regression models can be found in Fahrmeir et al. [5].

Looking at the example (1), and having the decompositions in mind, we can see that for "age" and "Apache II score" four different modeling possibilities exist. We can specify linear base-learners, one base-learner for the smooth deviation from linearity, a linear time-varying effect and a smooth deviation from linearity for this time-varying effect. The categorical covariate "fungal infection" has one possibility less. Nonlinear effects are not applicable for this kind of variables but linear effects, linear time-varying effects and nonlinear, time-varying effects can obviously be constructed and interpreted.

One should add that the clear separation and straightforward interpretation of the resulting selections and effects get lost if one adds the decomposition of $f_{\text {smooth }}(x)$ and at the same time the decomposition of $f_{\text {smooth }}(t) \cdot x$ to the model. Thus, we could get linear terms, polynomial terms, and smooth terms for $x$ as well as interactions of $x$ with a linearly, polynomially, and smoothly added $t$. With this many possible base-learners, interpretation is at least tricky. However, component-wise boosting has been shown to lead to sparse models and thus is especially useful in high-dimensional settings. Variable selection and model choice is even possible in data sets with $n \ll p$. Moreover, as in each iteration only one base-learner is fitted, boosting is capable to include more base-learners than observations in the data set.

\subsection{Likelihood-Based Boosting for Survival Data (CoxFlexBoost)}

The boosting algorithm, which we will present in the following section, is essentially based on the likelihood-based boosting approach as proposed by Tutz and Binder [20]. As we specially focus on the inclusion of flexible and time-varying terms in Cox-type additive models, we call the new algorithm CoxFlexBoost.

In the following, we denote the $j$-th base-learner by $g_{j}\left(\boldsymbol{x}(t) ; \boldsymbol{\beta}_{j}\right), j=1, \ldots, J$, where $J$ is the number of base-learners (possibly after decomposing nonlinear effects into several separate base-learners as described in the previous section). The base-learner can be seen as a generic representation for different types of functions. The covariates $\boldsymbol{x}(t)$ include classical covariates and possible time-varying effects expressed as artificial time-dependent covariates or the time $t$ itself. The notation $\boldsymbol{x}(t)$ for the covariates stresses the possible dependence on time. Thus, $g_{j}\left(\boldsymbol{x}(t) ; \boldsymbol{\beta}_{j}\right)$ can correspond to a linear function of $\tilde{x}$, where $\tilde{x}$ is a covariate from $\boldsymbol{x}(t)$, or of time $t$, with $t$ being the time covariate from $\boldsymbol{x}(t)$, 
or, more flexible, a smooth function of $\tilde{x}$ or $t$. Moreover, time-varying effects, expressed as varying coefficients, can be represented by the generic base-learner $g_{j}\left(\boldsymbol{x}(t) ; \boldsymbol{\beta}_{j}\right)$, where the effect of time $t$ is either a linear or a flexible function. With this notation at hand, we can derive the CoxFlexBoost algorithm:

\subsubsection{CoxFlexBoost Algorithm}

(i) Initialization: Set the iteration index $m:=0$.

a) Initialize the function estimates

$$
\hat{f}_{j}^{[0]}(\cdot) \equiv 0 .
$$

b) Initialize the additive predictor $\hat{\eta}^{[0]}$ with the maximizer of the log-likelihood of the intercept model, as offset value, i.e., with the maximum likelihood estimate for a constant log-hazard:

$$
\hat{\eta}^{[0]}(\cdot) \equiv \log \left(\frac{\sum_{i=1}^{n} \delta_{i}}{\sum_{i=1}^{n} t_{i}}\right)
$$

(ii) Estimation: Increase $m$ by 1. Fit all (linear and/or P-spline) base-learners

$$
\hat{g}_{j}(\cdot)=g_{j}\left(\cdot ; \hat{\boldsymbol{\beta}}_{j}\right), \forall j \in\{1, \ldots, J\},
$$

determined by penalized maximum likelihood estimation

$$
\hat{\boldsymbol{\beta}}_{j}=\underset{\boldsymbol{\beta}_{j}}{\operatorname{argmax}} l_{\mathrm{pen}, j}^{[m]}\left(\boldsymbol{\beta}_{j}\right),
$$

with the penalized log-likelihood (cf. Eq. (5))

$$
\begin{aligned}
l_{\text {pen }, j}^{[m]}\left(\boldsymbol{\beta}_{j}\right)= & \sum_{i=1}^{n}\left[\delta_{i} \cdot\left(\hat{\eta}_{i}^{[m-1]}\left(\boldsymbol{x}_{i}\left(t_{i}\right)\right)+g_{j}\left(\boldsymbol{x}_{i}\left(t_{i}\right) ; \boldsymbol{\beta}_{j}\right)\right)\right. \\
& \left.\quad-\int_{0}^{t_{i}} \exp \left\{\hat{\eta}_{i}^{[m-1]}\left(\boldsymbol{x}_{i}(\tilde{t})\right)+g_{j}\left(\boldsymbol{x}_{i}(\tilde{t}) ; \boldsymbol{\beta}_{j}\right)\right\} d \tilde{t}\right] \\
& -\operatorname{pen}_{j}\left(\boldsymbol{\beta}_{j}\right),
\end{aligned}
$$

where $\operatorname{pen}_{j}\left(\boldsymbol{\beta}_{j}\right)=\kappa_{j} / 2 \cdot \boldsymbol{\beta}_{j}^{\top} \boldsymbol{K}_{j} \boldsymbol{\beta}_{j}$ is the difference penalty for the $j$-th base-learner, or $\operatorname{pen}_{j}\left(\boldsymbol{\beta}_{j}\right)=$ 0 if the corresponding base-learner is unpenalized (i.e., here: a linear base-learner).

(iii) Selection: Choose the base-learner $\hat{g}_{j^{*}}$ that maximizes the unpenalized log-likelihood (cf. Eq. (11) with $\left.\operatorname{pen}_{j}(\cdot) \equiv 0\right)$

$$
j^{*}=\underset{j \in\{1, \ldots, J\}}{\operatorname{argmax}} l_{j}^{[m]}\left(\hat{\boldsymbol{\beta}}_{j}\right),
$$

where

$$
\begin{aligned}
l_{j}^{[m]}\left(\hat{\boldsymbol{\beta}}_{j}\right)=\sum_{i=1}^{n} & {\left[\delta_{i} \cdot\left(\hat{\eta}_{i}^{[m-1]}\left(\boldsymbol{x}_{i}\left(t_{i}\right)\right)+g_{j}\left(\boldsymbol{x}_{i}\left(t_{i}\right) ; \hat{\boldsymbol{\beta}}_{j}\right)\right)\right.} \\
& \left.-\int_{0}^{t_{i}} \exp \left\{\hat{\eta}_{i}^{[m-1]}\left(\boldsymbol{x}_{i}(\tilde{t})\right)+g_{j}\left(\boldsymbol{x}_{i}(\tilde{t}) ; \hat{\boldsymbol{\beta}}_{j}\right)\right\} d \tilde{t}\right]
\end{aligned}
$$

(iv) Update: 
a) Compute the update for the function estimate of the selected base-learner

$$
\hat{f}_{j^{*}}^{[m]}(\cdot)=\hat{f}_{j^{*}}^{[m-1]}(\cdot)+\nu \cdot \hat{g}_{j^{*}}(\cdot)
$$

and set $\hat{f}_{j}^{[m]}(\cdot)=\hat{f}_{j}^{[m-1]}(\cdot)$ otherwise (i.e., for $j \neq j^{*}$ ).

b) Compute the update for the additive predictor

$$
\hat{\eta}^{[m]}(\cdot)=\hat{\eta}^{[m-1]}(\cdot)+\nu \cdot \hat{g}_{j^{*}}(\cdot) .
$$

We choose the step-length factor $\nu=0.1$ but, in general, it is sufficient to choose $\nu \in(0,1]$ small enough.

(v) Stopping rule: Continue iterating steps (ii) to (iv) until $m=m_{\text {stop }}$.

Note that the estimated additive predictor from the previous iteration is treated as an offset in the first part of the formulas (11) and (12) and it is possibly time-dependent in the integral. The term $\hat{\eta}_{i}^{[m-1]}\left(\boldsymbol{x}_{i}(\tilde{t})\right)$ in the integral can be interpreted in such a way that the estimated parameters and the time-constant covariates of the base-learners are kept fixed and the time $\tilde{t}$ stays variable. Hence, the integrand in (11) is a function depending on the coefficient $\boldsymbol{\beta}_{j}$, which we try to estimate, and (possibly) on time $\tilde{t}$. In (12), we use the estimates $\hat{\boldsymbol{\beta}}_{j}$ from step (ii). Thus, we only have a function that (possibly) depends on time $\tilde{t}$.

A crucial tuning parameter in (component-wise) boosting is the stopping iteration $m_{\text {stop. }}$. As the base-learners are designed to be weak learners (i.e., only produce a slightly better estimate in each iteration) a small number of iterations corresponds to some kind of regularization (see, e.g., [21]). Furthermore, both variable selection and model choice are enforced by early stopping, as at most $m_{\text {stop }}$ different covariates (or model terms) can enter the model. Determining an optimal stopping iteration can be achieved with an information criterion (e.g., AIC, the corrected AIC [22] or the gMDL criterion [23]). However, Hastie [24] argues in favor of $k$-fold cross-validation (CV) to obtain the stopping iteration. As $\mathrm{CV}$ does not involve estimation of the degrees of freedom (which tend to underestimate the true degrees of freedom [24]) this is a more sensible solution. The only drawback one needs to mention here is the increased computational burden as the model needs to be estimated $k$ times.

In this paper, as we mainly focus on simulation studies, we use a validation data set to compute the (unpenalized) log-likelihood criterion (i.e., (5) without penalty). An appropriate stopping iteration is determined as the number of boosting iterations $\widehat{m}_{\text {stop,opt }}$ that maximizes the log-likelihood on the validation data.

\subsubsection{Remarks on Computational Considerations}

We have to integrate over time $\tilde{t}$ for each base-learner, in each boosting iteration and in each step of the optimization method (in our implementation the Broyden-Fletcher-Goldfarb-Shanno [BFGS] method, see, e.g., [25]) used to determine $\hat{\boldsymbol{\beta}}_{j}$. Hence, the estimation step (ii), or more precisely the integrations therein, are the computational bottleneck of the algorithm. By accelerating the integration method we have been able to increase the speed of CoxFlexBoost dramatically. However, further accelerations are possible.

In the following enumeration, we want to discuss some of the important computational issues and considerations that arose in CoxFlexBoost:

(a) Tutz and Binder [20] use a one-step Fisher scoring estimate in their likelihood-based boosting approach for each base-learner in each boosting iteration. Instead of this estimate, we use a full maximum likelihood estimate and apply a step-length factor $\nu$ as proposed in the FGD boosting 
literature (e.g.,[11]). This can possibly be computationally more intensive but we get an estimate that is "weakened" or "shrunken" with the same relative amount $\nu$ for all elements of the coefficient vector of the base-learner. Different amounts of shrinkage for one-step Fischer scoring may especially occur when competing base-learners with different numbers of parameters are used (e.g., linear base-learners vs. P-spline base-learners). This might result in a biased selection of (competing) model terms.

(b) Tutz and Binder [20] specify the smoothness of the base-learners using the smoothing parameter $\kappa$. They propose to choose $\kappa$ very large in order to obtain a weak learner (as desired for boosting). Only one single smoothing parameter is used for all base-learners and is chosen relatively crude. However, we believe that specifying the degrees of freedom df to determine the amount of smoothness of each base-learner (separately) is much more intuitive. Especially when model choice should be integrated in the boosting algorithm, we need to be able to define each base-learner in such a way that its complexity (in terms of df) is comparable to that of other model terms.

To specify the smoothing parameters via the corresponding degrees of freedom we exploit the relation that the latter depend on $\kappa_{j}$ and thus we can solve

$$
\operatorname{df}\left(\kappa_{j}\right)-\widetilde{d f}_{j} \stackrel{!}{=} 0
$$

for $\kappa_{j}$ with a pre-specified value of $\widetilde{d f}_{j}$. However, as the degrees of freedom in survival models are defined as

$$
\operatorname{df}\left(\kappa_{j}\right)=\operatorname{trace}\left[\boldsymbol{F}_{j}^{[m]}\left(\boldsymbol{F}_{j}^{[m]}+\kappa_{j} \boldsymbol{K}_{j}\right)^{-1}\right]
$$

(see Eq. (8)) we cannot solve the equation directly: The Fisher matrix of the base-learner $j$ in the $m$-th boosting iteration $\boldsymbol{F}_{j}^{[m]}$ depends on the design matrix and, at the same time, through the hazard rate $\lambda(\cdot)=\exp \left(\hat{\eta}^{[m-1]}(\cdot)+g_{j}\left(\cdot ; \boldsymbol{\beta}_{j}\right)\right)$ on the coefficients $\boldsymbol{\beta}_{j}$. Hence, the estimated degrees of freedom (14) do not only depend on the design matrix, the order of the penalty and the smoothing parameter $\kappa_{j}$ but also on the coefficients $\boldsymbol{\beta}_{j}^{[m]}$ of the $m$-th boosting iteration. We want to compute the smoothing parameters $\kappa_{j}, j=1, \ldots, J$ that correspond to the specified initial degrees of freedom $\widetilde{d f}_{j}$ in advance of the first boosting iteration, when no estimates of $\boldsymbol{\beta}_{j}$ are available. Hence, we set $\boldsymbol{\beta}_{j}^{[0]}:=0$ in (14) and solve (13) for $\kappa_{j}$ for each base-learner $g_{j}(\cdot ; \cdot), j=1, \ldots, J$.

(c) Another problem that emerges for likelihood-based boosting is that the specification of a constant smoothing parameter $\kappa_{j}$ for the base-learner $g_{j}\left(\cdot, \boldsymbol{\beta}_{j}\right)$ does not correspond to a fixed amount of smoothness for this base-learner. With an increasing number of iterations $m$, the degrees of freedom df ${ }_{j}^{[m]}$ for $g_{j}\left(\cdot, \boldsymbol{\beta}_{j}\right)$ change, as we could see in our simulation studies (results not presented here). However, this effect is not very strong. Over numerous boosting iterations $m$, only minor changes of the estimated degrees of freedom $\mathrm{df}_{j}^{[m]}$ of the $j$-th base-learner are observed. Thus we propose to use the above approximation of degrees of freedom and to ignore the (small) changes with increasing iterations. Thinking of a correction, we could readjust the smoothing parameter $\kappa_{j}$ in each (or each $k$-th) iteration such that we get again the desired degrees of freedom. However, this would lead to an increased computational burden. As we could observe only minor deviations and as the degrees of freedom (8) are just an approximation themselves, readjusting $\kappa_{j}$ does not seem to be necessary.

We can see from (b) that we are able to use initial degrees of freedom to get an approximate value for $\kappa_{j}$. Even if we may have a slight misspecification, this is more intuitive than defining the smoothing parameter itself. Moreover, this allows us to use the model choice scheme as proposed by Kneib et al. [12]. As the problem of changing degrees of freedom (c) is not that strong, the different model terms stay roughly comparable even in larger boosting iterations. In the next section, we want to support these statements with simulation studies. 


\section{Simulations and an Application}

\subsection{Simulations}

\subsubsection{Outline of Simulations}

To gain deeper insights in the properties of the proposed CoxFlexBoost procedure, two simulation studies were performed. In both settings, we generated data sets consisting of 300 observations in the learning sample and 100 observations in the validation sample. The former sample was used to fit a structured survival model (2) with CoxFlexBoost, and the latter to determine the stopping iteration $m_{\text {stop }}$. The survival data was simulated applying a generalization of the algorithm proposed by Bender et al. [26]. They propose a flexible framework to sample survival times for Cox proportional hazards models, which can be extended to sample survival data with time-varying effects.

In the first setting, data was simulated without any time-varying effects. Even the baseline hazard was chosen constant over time. This corresponds to data from an exponential distribution (given the covariate values $\boldsymbol{x}$ ). In this setting, the goal was to evaluate the performance of the algorithm with respect to the detection of linear and smooth effects applying CoxFlexBoost with the decomposition for model choice as given in Section 3.1. Another interesting topic was the investigation of the ability to perform variable selection, i.e., the ability of the algorithm to leave the non-effective covariates unselected. A covariate is not selected if none of the model terms that include this covariate is chosen.

The second setting included time-varying effects for the baseline hazard and a categorical covariate. This corresponds to different baseline hazards in the two groups. We tried to investigate, whether the algorithm selects time-varying effects even if there are none present, whether the time-varying effect was detected correctly and whether it was appropriately estimated. Furthermore, we investigated the properties of variable selection and wanted to check if other effects, as linear and smooth effects, are detected and modeled "correctly".

Computational Details The simulations were conducted using R [27]. The proposed CoxFlexBoost algorithm is implemented in an add-on package CoxFlexBoost [28]. The main function to fit structured survival models is called cfboost(). The syntax and usage is similar to the R package mboost [29] for model-based boosting, which implements a generic interface for functional gradient descent boosting. The data was simulated using the rSurvTime() function as given in the package CoxFlexBoost [28].

We will utilize linear or P-spline base-learners in the following. Per default, the inner knots of the P-splines are equally spaced covering the range of the corresponding covariate. We only use 20 (inner) knots, as increasing the number computationally is quite demanding and empirically little is gained regarding the prediction performance ([7]).

Details on Simulation Scheme 1 As already stated, we have two different simulation schemes. For the first study, we simulated 400 realizations of 15 i.i.d. covariates $X_{1}, \ldots, X_{15}$ according to

$$
\begin{array}{ccc}
X_{1}, X_{2}, & X_{7}, X_{8}, X_{9} & \stackrel{\text { i.i.d. }}{\sim} U[-1,1] \\
X_{3}, X_{4}, & X_{10}, X_{11}, X_{12} & \stackrel{i . i . d .}{\sim} N(0,1) \\
\underbrace{X_{5}, X_{6},}_{\text {effective covariates }} & \underbrace{X_{13}, X_{14}, X_{15}}_{\text {non-effective covariates }} & \stackrel{\text { i.i.d. }}{\sim} B(1,0.5) .
\end{array}
$$

The covariate realizations $\boldsymbol{x}_{i}=\left(x_{1, i}, \ldots, x_{15, i}\right), i=1, \ldots, 400$, were used to simulate survival times with the hazard rate

$$
\begin{aligned}
\lambda(t, \boldsymbol{x})=\exp (2 & +\sin \left(-x_{1}^{2}-0.6 x_{1}^{3}\right)+1.4 x_{2}^{2} \\
& \left.+0.5 \sin \left(1.5 x_{3}\right)+x_{4}-2 x_{5}+0.1 x_{6}\right)
\end{aligned}
$$


using the inversion method proposed by Bender et al. [26]. Only the covariates $X_{1}$ to $X_{6}$ have an effect on the survival time. We call these covariates "effective covariates". Covariates $X_{7}$ to $X_{15}$ have no effect on the sampled times. Therefore, we use the term "non-effective covariates" for these variables. We have two uniformly distributed, two standard normally distributed and two binary distributed covariates in the model. $X_{1}$ to $X_{3}$ have nonlinear effects, $X_{4}$ and the categorical variables $X_{5}$ and $X_{6}$ have linear effects. The effects are depicted in Figure 1 . The censoring times $C_{i}$ are simulated i.i.d. exponentially distributed with rate $\lambda=1 / \bar{t}$, i.e., with $\mathbb{E}(C)=\frac{1}{n} \sum_{i=1}^{n} t_{i}=\bar{t}$, leading to a non-censoring rate of approximately $70 \%$. Table 1 (upper part) gives an overview of the covariates and the way they were allowed to enter the model. In the first setting, this can be either as linear base-learner or as P-spline base-learner.

In the table we denote the base-learners with the names of the R-functions in CoxFlexBoost. The function bols () creates a linear base-learner and bbs () represents a P-spline base-learner. bolsTime() and bbsTime() are functions for linear and P-spline base-learners of time. Both base-learners of time represent time-varying effects expressed as varying coefficient terms, i.e., if a covariate (other than time) is associated with these base-learners, it is modeled as an interaction of time (as linear or smooth term) with the respective covariate. Furthermore, the initial degrees of freedom are given in brackets for flexible base-learners. Note that we set the initial degrees of freedom $\mathrm{df}=1$ and centered the function (see Sec. 3.1).

Details on Simulation Scheme 2 In the second simulation scheme we included a time-dependent baseline hazard. Additionally, one time-varying effect was specified. Model choice, based on the decompositions (9) and (10), was performed. To reduce the computational burden, we decided to include only effective covariates. Thus, we sampled the six covariates $X_{1}$ to $X_{6}$ according to

$$
\begin{aligned}
& X_{1}, X_{2}, \stackrel{\text { i.i.d. }}{\sim} U[-1,1] \\
& X_{3}, X_{4}, \quad \stackrel{i . i . d .}{\sim} N(0,1) \\
& \underbrace{X_{5}, X_{6}}_{\text {effective covariates }}, \stackrel{\text { i.i.d. }}{\sim} B(1,0.5) \text {. }
\end{aligned}
$$

Applying the inversion method we used 400 realizations $\boldsymbol{x}_{i}=\left(x_{1, i}, \ldots, x_{6, i}\right), i=1, \ldots, 400$ to sample survival times with the hazard rate

$$
\begin{aligned}
\lambda(t, \boldsymbol{x})=\exp (2 & +\log (t+0.2)+\sin \left(-x_{1}^{2}-0.6 x_{1}^{3}\right)-0.3 x_{2} \\
& \left.+0.5 \sin \left(1.5 x_{3}\right)+x_{4}-2 x_{5}+2 \sqrt{t} \cdot x_{6}\right) .
\end{aligned}
$$

Like in the previous simulation scheme we simulated the censoring times $C_{i} \stackrel{i . i . d .}{\sim} \operatorname{Expo}(1 / \bar{t})$. In this case this corresponds to a non-censoring rate of approximately $50 \%$. The base-learners that were used in the model are given in Table 1 (lower part).

\subsubsection{Simulation Results 1: Model Choice and Variable Selection}

In the first scheme we simulated 200 randomly drawn replicates of the data set. Each data set was sampled with the hazard rate (16). In the second scheme with time-varying effects (18) the number of simulation replicates was 50 . We need to mention that it took about one day to estimate the model for one data set in the second scheme. The reason can be found in the high computational burden of the estimation of time-varying effects (see Sec. 3.2.2). For models without time-varying base-learners the integral (11) in the algorithm drastically simplifies, i.e., it becomes a simple product of the exponent of the additive predictor and the observed survival times, and thus estimation speeds up vastly. Another reason for the deceleration of the algorithm with time-varying effects is the increase 


\begin{tabular}{llcccc}
\hline \multicolumn{5}{c}{ Simulation Scheme 1 } \\
\hline & Type & bols & bbs $(\ldots$, df $=1)$ & bolsTime & bbsTime $(\ldots, \mathrm{df}=1)$ \\
\hline$x_{1}-x_{4}$ & continuous & $\checkmark$ & $\checkmark$ & & \\
$x_{5}-x_{6}$ & categorical & $\checkmark$ & & \\
$x_{7}-x_{12}$ & continuous & $\checkmark$ & $\checkmark$ & \\
$x_{13}-x_{15}$ & categorical & $\checkmark$ & & \\
\hline
\end{tabular}

\begin{tabular}{llcccc}
\hline \multicolumn{5}{c}{ Simulation Scheme 2 } \\
\hline & Type & bols & $\operatorname{bbs}(\ldots, \mathrm{df}=1)$ & bolsTime & bbsTime $(\ldots, \mathrm{df}=1)$ \\
\hline$t$ & time & & $\checkmark$ & $\checkmark$ & $\checkmark$ \\
$x_{1}-x_{4}$ & continuous & $\checkmark$ & $\checkmark$ & $\checkmark$ \\
$x_{5}-x_{6}$ & categorical & $\checkmark$ & & $\checkmark$ & $\checkmark$ \\
\hline
\end{tabular}

Table 1: Overview of combinations of covariates and base-learners in the two simulation schemes. Combinations with $\checkmark$ were used in the model formula.

of possible base-learners. Compared to a model where we do not allow for time-varying effects, the number of base-learners for a continuous covariate doubles and for categorical covariates even triples. As each of the base-learners needs to be fitted in every boosting iteration this has an enormous impact on speed.

Simulation Scheme 1 In the following section, we explore the accuracy of model choice (and thus of variable selection) given by the relative frequency of (correctly) selected base-learners. This means we count the models (simulation replicates) where the base-learner was included and ignore how often and in which boosting iteration(s) the base-learner was selected.

Table 2 (left) shows the selection frequencies of the base-learners in the first simulation scheme. Only the variables $x_{1}$ to $x_{6}$ have an effect on the hazard rate (16) and thus, on the survival time. These effective covariates are presented in the upper half of the table. We see that almost all effective base-learners have a selection frequency close to one or exactly one except for the linear base-learners bols $\left(x_{2}\right)$ and bols $\left(x_{6}\right)$. If we look at the true influence of $x_{2}$ (see (16)) it shows that this is a good result, as we have a quadratic influence of this covariate and hence no linear effect is required. The low selection frequency of bols $\left(x_{6}\right)$ can be attributed to the size of the effect of $x_{6}$, which is very small. It is 20 times smaller than the effect of the other categorical covariate $x_{5}$. Hence, the low selection frequency seems very plausible. For $x_{4}$ (which has in reality a linear effect) the algorithm selected in $23 \%$ of the replicates a flexible deviation from linearity. Thus, in some models the (wrong) impression of an underlying nonlinear effect of $x_{4}$ is given. However, compared to the selection frequencies of the other effects, this is only of minor importance. In addition, in Section 4.1.3 we will see that the departures from linearity are only very small.

In the lower part of Table 2 (left) we expect the selection frequency of a base-learner to be close to zero or at least substantially smaller than for effective covariates. When we look at the noneffective covariates we see that the frequencies of selection are much smaller than those of the effective covariates.

Note that the number of base-learners is not equal to the number of variables. A variable is selected if any of the base-learners of this variable is selected. Using this definition, we see that on average we selected 9.97 variables with 5.465 effective variables and 4.505 non-effective variables. Compared to a scheme where we assign only one base-learner for each variable (e.g., a flexible base-learner with 4 degrees of freedom) we realize that the model choice scheme tends to select more variables and to select more non-effective variables. Perhaps, this is due to an increased number of possible base-learners per covariate. This argument is backed by the finding that we selected 13 out of 25 base-learners. 
We selected (on average) about 5.5 non-effective base-learners which corresponded on average to 4.5 non-effective variables. Thus, almost every non-effective base-learner is based on another variable.

\begin{tabular}{lr}
\hline \multicolumn{2}{c}{ Simulation Scheme $\mathbf{1}$} \\
\hline \multicolumn{2}{c}{ Effective Covariates } \\
\hline $\operatorname{bols}\left(x_{1}\right)$ & 0.80 \\
$\operatorname{bbs}\left(x_{1}\right)$ & 0.97 \\
$\operatorname{bols}\left(x_{2}\right)$ & 0.29 \\
$\operatorname{bbs}\left(x_{2}\right)$ & 1.00 \\
$\operatorname{bols}\left(x_{3}\right)$ & 0.88 \\
$\operatorname{bbs}\left(x_{3}\right)$ & 0.90 \\
$\operatorname{bols}\left(x_{4}\right)$ & 1.00 \\
$\operatorname{bbs}\left(x_{4}\right)$ & $\mathbf{0 . 2 3}$ \\
$\operatorname{bols}\left(x_{5}\right)$ & 1.00 \\
$\operatorname{bols}\left(x_{6}\right)$ & 0.48 \\
\hline $\operatorname{Non}-\mathbf{E f f e c t i v e ~ C o v a r i a t e s}$ \\
\hline $\operatorname{bols}\left(x_{7}\right)$ & 0.36 \\
$\operatorname{bbs}\left(x_{7}\right)$ & 0.52 \\
$\operatorname{bols}\left(x_{8}\right)$ & 0.36 \\
$\operatorname{bbs}\left(x_{8}\right)$ & 0.46 \\
$\operatorname{bols}\left(x_{9}\right)$ & 0.38 \\
$\operatorname{bbs}\left(x_{9}\right)$ & 0.50 \\
$\operatorname{bols}\left(x_{10}\right)$ & 0.34 \\
$\operatorname{bbs}\left(x_{10}\right)$ & 0.28 \\
$\operatorname{bols}\left(x_{11}\right)$ & 0.32 \\
$\operatorname{bbs}\left(x_{11}\right)$ & 0.29 \\
$\operatorname{bols}\left(x_{12}\right)$ & 0.37 \\
$\operatorname{bbs}\left(x_{12}\right)$ & 0.24 \\
$\operatorname{bols}\left(x_{13}\right)$ & 0.41 \\
$\operatorname{bols}\left(x_{14}\right)$ & 0.36 \\
$\operatorname{bols}\left(x_{15}\right)$ & 0.32 \\
\hline &
\end{tabular}

\begin{tabular}{|c|c|}
\hline \multicolumn{2}{|c|}{ Simulation Scheme 2} \\
\hline \multicolumn{2}{|c|}{ Effective Covariates } \\
\hline $\operatorname{bolsTime}(t)$ & 0.52 \\
\hline $\operatorname{bbsTime}(t)$ & 0.92 \\
\hline $\operatorname{bols}\left(x_{1}\right)$ & 0.38 \\
\hline $\operatorname{bbs}\left(x_{1}\right)$ & 1.00 \\
\hline $\operatorname{bolsTime}\left(t, x_{1}\right)$ & 0.80 \\
\hline $\operatorname{bbsTime}\left(t, x_{1}\right)$ & 0.40 \\
\hline $\operatorname{bols}\left(x_{2}\right)$ & 0.34 \\
\hline $\operatorname{bbs}\left(x_{2}\right)$ & 0.60 \\
\hline $\operatorname{bolsTime}\left(t, x_{2}\right)$ & 0.94 \\
\hline $\operatorname{bbsTime}\left(t, x_{2}\right)$ & 0.26 \\
\hline $\operatorname{bols}\left(x_{3}\right)$ & 0.32 \\
\hline $\operatorname{bbs}\left(x_{3}\right)$ & 0.90 \\
\hline $\operatorname{bolsTime}\left(t, x_{3}\right)$ & 0.84 \\
\hline $\operatorname{bbsTime}\left(t, x_{3}\right)$ & 0.32 \\
\hline $\operatorname{bols}\left(x_{4}\right)$ & 0.98 \\
\hline $\operatorname{bbs}\left(x_{4}\right)$ & 0.24 \\
\hline $\operatorname{bolsTime}\left(t, x_{4}\right)$ & 1.00 \\
\hline $\operatorname{bbsTime}\left(t, x_{4}\right)$ & 0.28 \\
\hline $\operatorname{bols}\left(x_{5}\right)$ & 1.00 \\
\hline $\operatorname{bolsTime}\left(t, x_{5}\right)$ & 0.80 \\
\hline $\operatorname{bbsTime}\left(t, x_{5}\right)$ & 0.66 \\
\hline $\operatorname{bols}\left(x_{6}\right)$ & 1.00 \\
\hline $\operatorname{bolsTime}\left(t, x_{6}\right)$ & 1.00 \\
\hline $\operatorname{bbsTime}\left(t, x_{6}\right)$ & 0.44 \\
\hline
\end{tabular}

Table 2: Relative frequencies of the selection of the base-learners in the first simulation scheme (left, 200 replicates) and in the second simulation scheme (right, 50 replicates). In the left table, the upper half shows the base-learners for covariates that have an influence on the hazard rate, the lower half those without influence. The right table only consists of influential covariates. Wrongly assigned linear, smooth or time-varying effects are printed in bold face.

Simulation Scheme 2 From Table 2 (right) we see that the second simulation with a timedependent baseline hazard rate and one time-varying effect shows a selection bias in favor of timevarying effects. We realize that some falsely selected base-learners for time-varying effects have selection frequencies close to one. These effects are included in the models as time-varying effects deliver some of the flexibility the model terms require. This problem is for example discussed in Therneau and Grambsch [18]. However, in most cases the true effects are also selected to enter the model and the selection frequency of the true effects is typically (slightly) higher than or at least comparable to the selection frequency of the time-varying effects.

The time-varying effect of $x_{6}$ is always discovered and the $(\log )$ baseline hazard is almost always selected. Although the time-varying effect of $x_{6}$ is in truth nonlinear, only in roughly half of the 
models a flexible time-varying effect is chosen.

Another problem that arises in this context is that we hardly can interpret the resulting effects as we almost always have a mixture of different modeling possibilities for the covariates in the model: $x_{1}$, for example, is selected as smooth effect (linear and centered smooth effect) and as (flexible) time-varying effect at the same time. Thus, the models are not really interpretable and mostly useful in the context of prediction.

\subsubsection{Simulation Results 2: Estimated Effects}

Now, we look at the estimated effects and compare them with the real, specified effects. To keep the plots clear and readable we restricted the results to 20 models for each simulation scheme.

Simulation Scheme 1 In Figure 1, we see a selection of the estimated effects for the six effective covariates. Note that all function estimates and all true effects are centered such that their mean is equal to zero. This is required, as the "level" of the estimates can be altered: In each base-learner we have parameters for the intercept to allow the overall estimate to reach the right level. Hence, the intercept estimate of a base-learner is not connected with the effect of the corresponding covariate and thus the "level" of a base-learner is generally arbitrary. Actually we only want to compare the form of the estimated effects.

The effects of $x_{1}$ to $x_{5}$ are estimated reasonable well. A selection of estimated effects can be found in Figure 1 whereas the other effects are comparable to the depicted ones. The estimated effects of $x_{6}$ seem to have a larger bias but if we take the scale of the outcome (i.e., the log hazard rate) into account we see that there is no big deviation.

Caused by the sparse data at the boundaries (note that we used a standard normal distribution to simulate $x_{3}$ ) the boundaries of the sine form of $x_{3}$ are estimated quite poorly, whereas the middle part is estimated quite sensible (not depicted here). For linear effects, the sparse tails do not pose such a big problem as we see from $x_{4}$. Only in some cases $(23 \%$, see Tab. 2 (left)) we have deviations from linearity. The estimation in the center region is hardly effected and only slight deviations in the areas with less observations can be identified. Hence, linear effects seem to be hardly effected by sparse tails.

The estimated effects of the non-effective covariates $x_{7}$ to $x_{15}$ (not depicted here) are more or less oscillating around zero (if present). Again the normally distributed variables show a higher variation at the boundaries. Categorical variables, which are seldom selected (see Tab. 2 (left)), show the smallest deviations from "no effect".

Simulation Scheme 2 Simulation scheme two has only effective covariates in the model formula. The focus is on the goodness of the estimation of time-varying effects and on the performance of the simultaneous model choice algorithm. In Section 4.1.2 we showed that CoxFlexBoost leads to a biased model choice in favor of time-varying effects. Note that for the plots in Figure 3 we did not take into account that the boosting procedure also selected time-varying effects for many covariates. Only the time-fixed effects are depicted except for the baseline hazard (see Fig. 2).

In the second simulation scheme, a time-dependent baseline hazard is added as well as a timevarying effect. The left panel of Figure 2 depicts the estimated log-baseline hazard over time in 20 models. Until time $t \approx 1$ the curvature of the true effect is fairly well estimated. Thereafter, the quality of the estimation rapidly decreases. This is due to the sparseness of the data as discussed above for normally distributed data. As it can be seen from the right graphic in Figure 2 the survival time has a sparse right tail which leads to unstable estimations as already pointed out by Gray [7].

Figure 3 shows a selection of the estimated effects, which are in some cases almost as good as in the first simulation but they all tend to be a bit more unstable. Especially the estimated effects of the second covariate $\left(x_{2}\right)$ show big deviations from the true, linear function. The time-varying effect of $x_{6}$ 

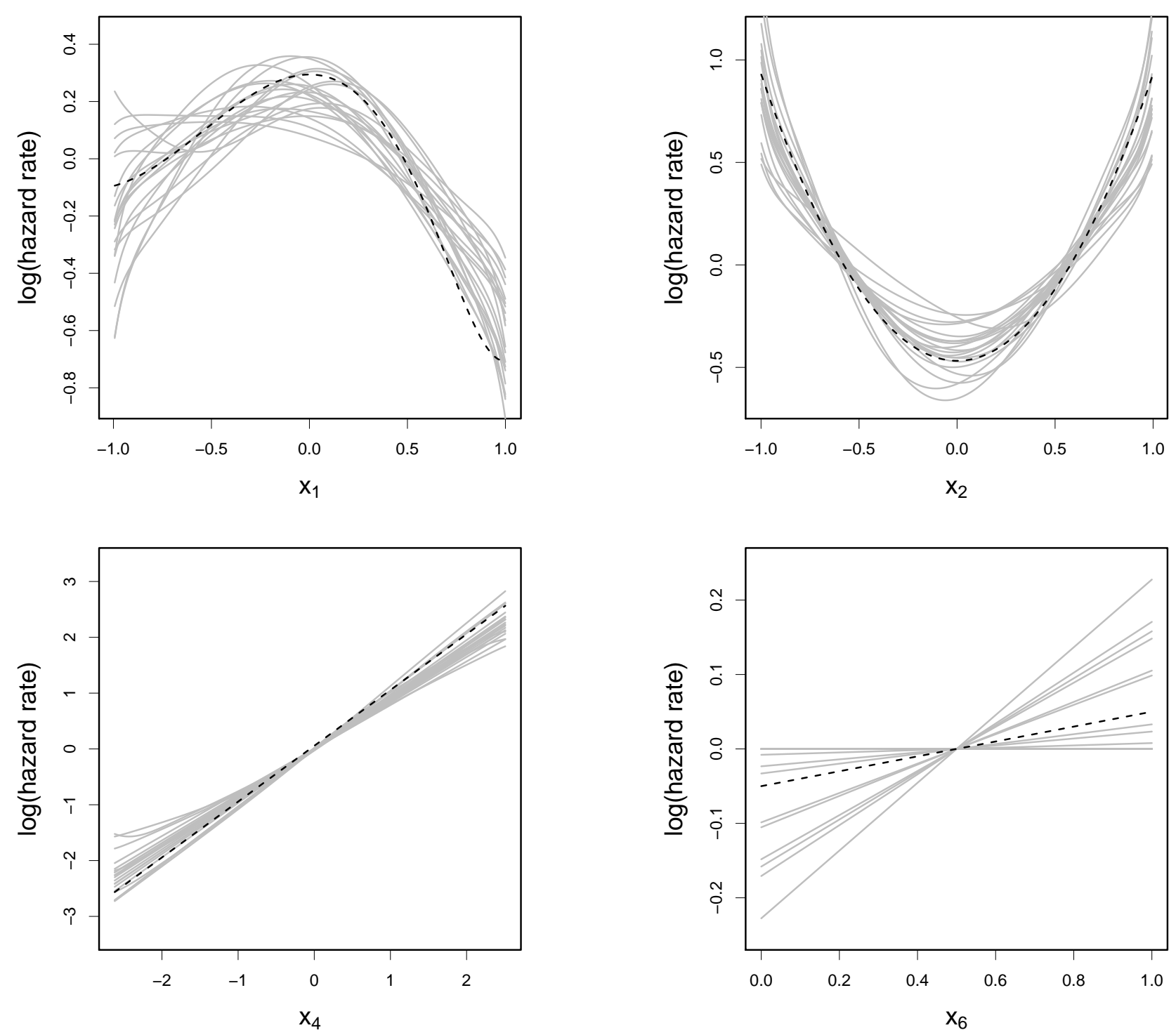

Figure 1: Simulation Scheme 1 - Estimation of covariate effects from 20 models (gray lines) and real effects (dashed lines). Effect estimates and real effects are centered.

(not depicted here) suffers from the same problem as the baseline hazard, i.e., the estimated function is very unstable in the sparse, right tail.

\subsection{Application: Model for Surgical Patients}

In the following section, we aim to build a model for patients with severe sepsis. Our retrospective analysis used data from a database, which was initiated in 1993 in the surgical intensive care unit, Department of Surgery, Klinikum Großhadern, Ludwig-Maximilians-Universität München, Germany, for local benchmarking and quality control. The documentation period started on March 1st, 1993, and lasted until February 28th, 2005. During this time, 5,079 patients (5,495 cases) were admitted to the intensive care unit. Baseline characteristics and detailed outcomes of that population were published recently $[30,31,32]$. A retrospective search of all eligible cases was conducted, where only cases that had to be treated because of severe sepsis were included. Patients likely to die of serious comorbid conditions (e.g., tumor progress) other than sepsis within the 90-day follow-up period were excluded 

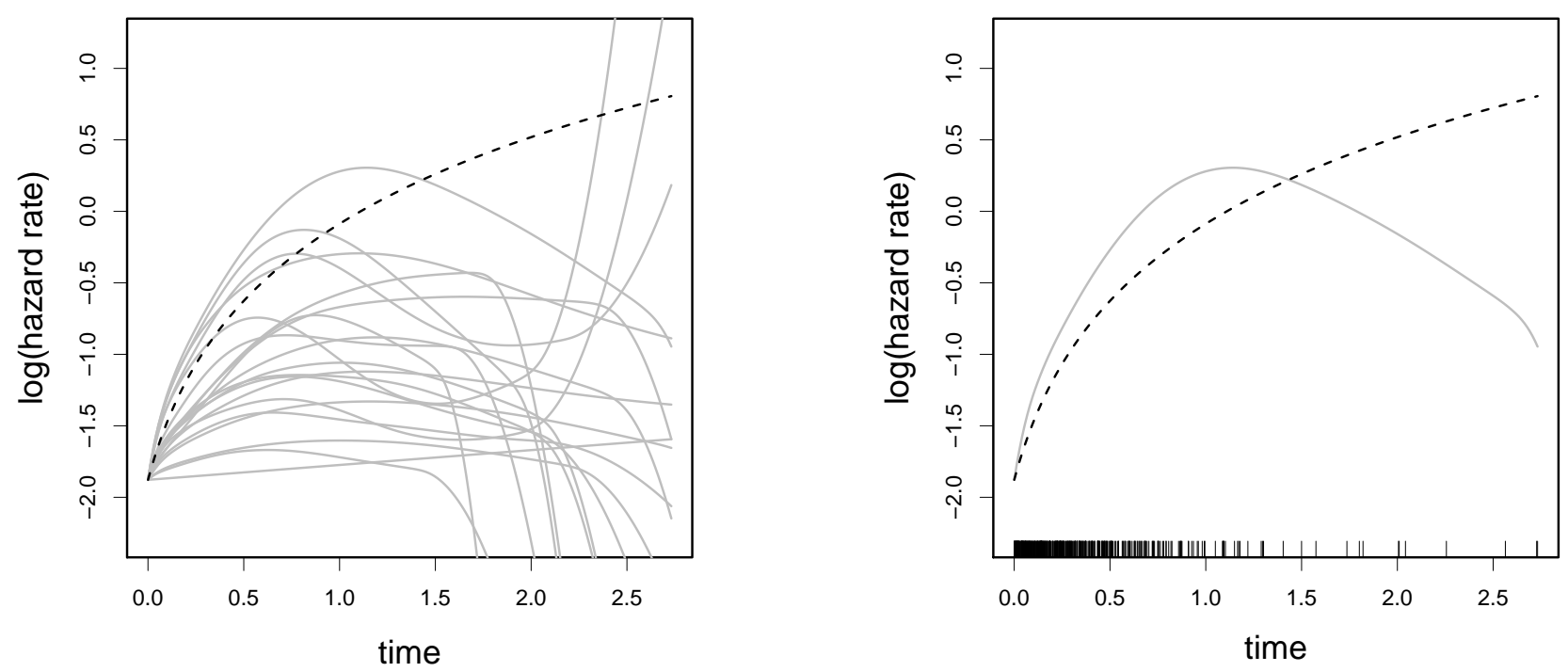

Figure 2: Simulation Scheme 2 - Left: Estimation of the baseline hazard from 20 models (gray lines) and real effect (dashed line). Right: Estimation of the baseline hazard for one model (gray line) and real effect (dashed line) together with rugs for the observed data. Offsets of effect estimates and real effects are set equal and the effects are centered.

from the analysis. Further inclusion criteria had to be met [2]. We obtained relevant covariates reflecting the state of the patient on admission day, and the 90-day survival time for 462 patients with severe sepsis. To build the model, we applied the proposed CoxFlexBoost algorithm to the data.

\subsubsection{Application of CoxFlexBoost}

To asses the stability of the variable selection and model choice process of component-wise boosting, as implemented in CoxFlexBoost [28], we used 5 random subsamples, each with 362 observations, of the severe sepsis data from Großhadern. The remaining 100 observations from each subsample were used to determine the stopping iteration.

Before entering the model, all continuous covariates except time were standardized on intervals $\left[\frac{x_{\min }}{x_{\max }-x_{\min }}, \frac{x_{\max }}{x_{\max }-x_{\min }}\right]=\left[\frac{x_{\min }}{x_{\max }-x_{\min }}, \frac{x_{\min }}{x_{\max }-x_{\min }}+1\right]$, where $x_{\min }$ and $x_{\max }$ are the minimum and maximum of the respective covariate. This was done by dividing by the range of the covariate:

$$
\widetilde{x_{i}}=\frac{x_{i}}{x_{\max }-x_{\min }} .
$$

Categorical covariates are dummy coded. Time enters the model unstandardized.

As we have realized in the simulation studies, it seems that boosting with model choice is unstable (w.r.t. the selected base-learners) and prefers time-varying base-learners.

The CoxFlexBoost algorithm is applied to the same data which has also been used in Hofner et al. [3], where another model selection strategy called two-stage stepwise (TSS) procedure is proposed for models with potentially time-varying effects. This makes it possible to directly compare the two methods. Thus, we want to look at the variable selection capabilities of both methods. The two-stage stepwise models were fitted with the software package BayesX (Vers. 1.51), which is freely available from http://www. stat.uni-muenchen.de/ bayesx [33].

In contrast to the two-stage stepwise procedure, CoxFlexBoost is not able to handle preset covariates. Such an approach could be included in the boosting framework, for example, by updating a set of mandatory covariates in every iteration (see, e.g., [34]). However, as this is not implemented in CoxFlexBoost so far, we did not use mandatory covariates but treated all covariates equal in the model 

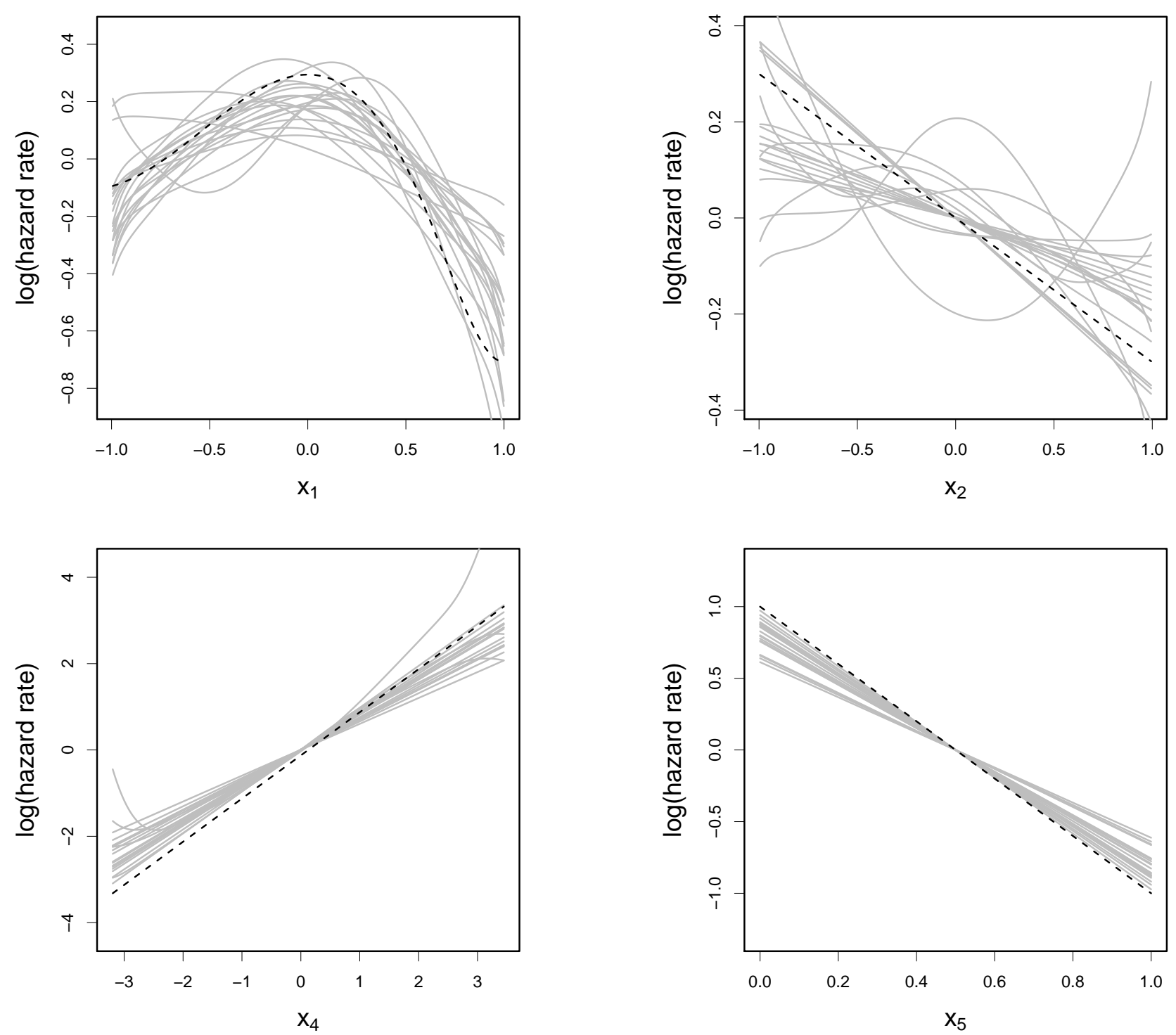

Figure 3: Simulation Scheme 2 - Estimation of covariate effects from 20 models (gray lines) and real effects (dashed lines). Effect estimates and real effects are centered.

choice procedure. In contrast, in the application of the TSS procedure [3] six mandatory covariates were used. This potentially can affect the inclusion of further covariates heavily. Furthermore, we did not use the complete data set but just subsamples in order to estimate the stopping iterations based on the out-of-bag sample. This again may have an influence on the selection and estimation of base-learners.

We extracted the selection frequencies for all variables in the CoxFlexBoost approach. A comparison with the model from the TSS approach can be found in Table 3.

We see that there is a fair range of agreement between CoxFlexBoost and the TSS procedure. To asses the disagreement, one needs to keep in mind that we used mandatory covariates in the TSS model, which perhaps would not have been added if a stopping criterion would have been applied. Both, "malignant primary disease" and "sex" were included in the starting model that consisted of the mandatory covariates despite they could not improve the conditional AIC. In the CoxFlexBoost model "renal replacement therapy" and "surgery for thoracic disease" were added only one or two times, respectively. In two-stage stepwise model they were added as the last two variables. This could 


\begin{tabular}{lcc}
\hline & \multicolumn{2}{c}{ Selected } \\
\cline { 2 - 3 } Covariate & CoxFlexBoost & Two-Stage Stepwise \\
\hline systolic blood pressure & 0 & not selected \\
readmission & 0 & not selected \\
direct postoperative admission & 0 & not selected \\
pneumonia & 0 & not selected \\
Horowitz ratio & 0 & selected \\
\hline malignant primary disease & 1 & mandatory \\
sex & 1 & mandatory \\
hemoglobin concentration & 1 & selected \\
renal replacement therapy & 1 & selected \\
\hline surgery for thoracic disease & 2 & selected \\
\hline emergency admission & 4 & not selected \\
creatinine concentration & 4 & selected \\
peritonitis & 4 & selected \\
\hline need for artificial ventilation & 5 & not selected \\
Apache II score & 5 & mandatory \\
palliative operation & 5 & mandatory \\
age & 5 & mandatory \\
treatment period & 5 & mandatory \\
fungal infection & 5 & selected \\
catecholamine therapy & 5 & selected \\
\hline
\end{tabular}

Table 3: Selection of Covariates: Comparison of CoxFlexBoost and the two-stage stepwise procedure. For CoxFlexBoost the number of models in which the covariate was selected is given (max. 5).

indicate that the inclusion of these covariates (in the TSS model) is at least arguable. "Horowitz ratio" and "hemoglobin concentration" were considered to be influential in the TSS model based on the conditional AIC. However, further inspection revealed that both effects only marginally depart from the zero-line, which would indicate that there is no effect at all. This is again in line with the results from CoxFlexBoost. "Need for artificial ventilation" and "emergency admission" were not included in the TSS model. CoxFlexBoost instead selected these variables as time-varying effects. As both variables have just a relatively small linear time-varying effect (see Fig. 4) these effects could be artifacts as well. Defining an inclusion rate of 2 or less negligible, only 10 out of 20 covariates can be regarded as influential covariates in the boosting model. The TSS procedure selected 14 covariates but 6 of these covariates were mandatory. Thus, a candidate model without a set of compulsory covariates could lead to a sparser final model. We can conclude that both the TSS procedure and CoxFlexBoost have a comparable strength for variable selection.

The resulting effects of the CoxFlexBoost models are hardly interpretable as many covariates are included with different modeling possibilities. They are added as smooth effects as well as time-varying effects. In Figure 4 the time-varying effects of four categorical covariates are depicted for two of the five estimated models. These two plots resemble the two archetypes of observed structures for the estimates of time-varying effects. Three models have the same structure as the model depicted in the left panel and the other two models have the same structure as depicted in the right plot. We only plotted the covariates that were selected in the majority of the five models. We could see that the log-baseline hazard is only selected in 3 out of the 5 models. Furthermore, it is remarkable that almost all time-varying effects were added as linear base-learners. Only observations in the subgroup 
with "fungal infection" have approximately a quadratic log-hazard rate. The log-hazard in the other subgroups does not substantially differ from the log-baseline hazard of the model without an additional time-varying effect (Fig. 4, solid line), i.e., hardly any time-varying effect for these covariates can be observed. This is consistent in all five models.
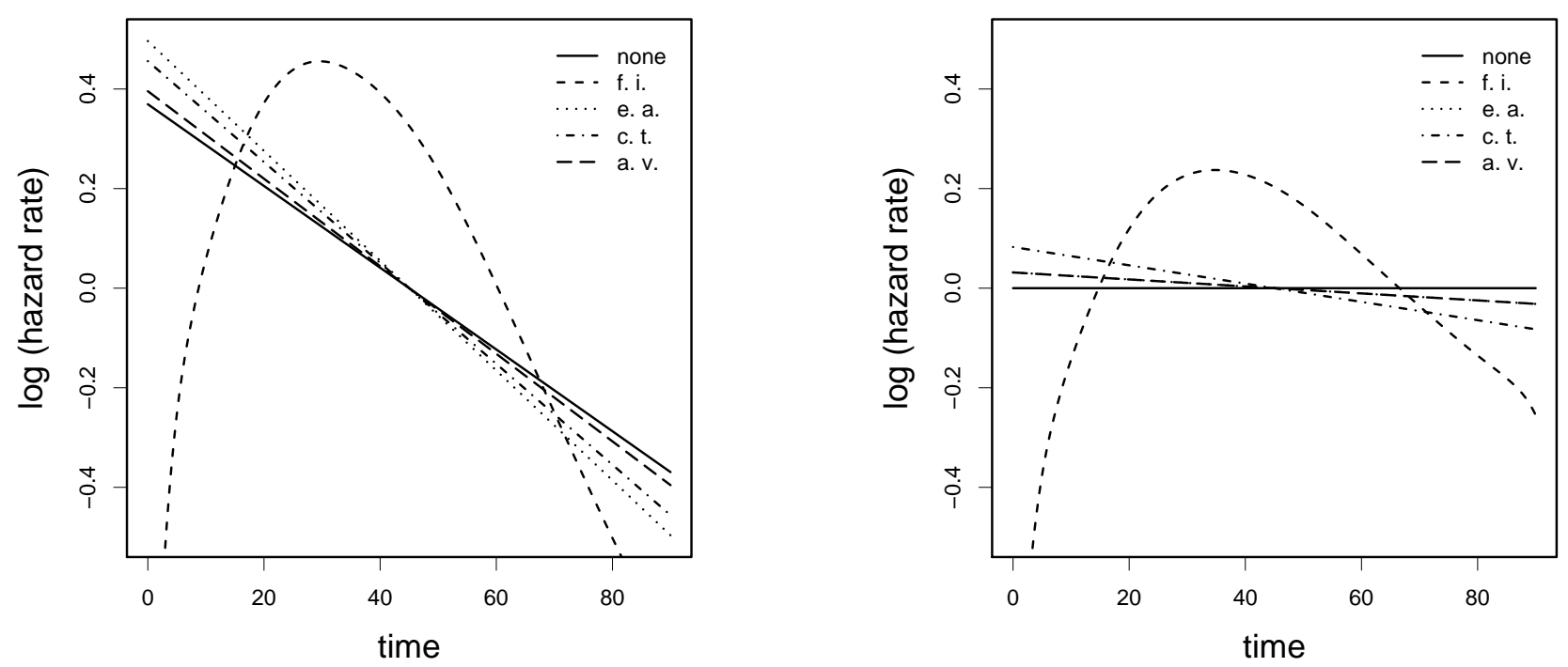

Figure 4: CoxFlexBoost with model choice procedure for surgical patients data in 2 (out of 5) subsamples: $\log$ (baseline hazard rate) in subgroups defined by "fungal infection" (f. i., present vs. absent), "emergency admission" (e. a.), "catecholamine therapy"(c. t.) and "artificial ventilation"(a. v.). All effects are centered.

Continuous covariates entered the model standardized. For some of the covariates, time-varying effects were also selected. Additional to the four categorical covariates with time-varying effect, six covariates were frequently added to the model: Three continuous covariates "Age", "Apache II score" and "creatinine concentration" and three categorical covariates "palliative operation", "peritonitis" (present vs. absent) and "treatment period" (before vs. after 2002). All three continuous covariates have a very high selection frequency for flexible time-varying effects. "Apache II score", for example, was added as a strong nonlinear effect to the TSS model, whereas CoxFlexBoost estimated only a linear effect but added an additional time-varying effect. This increased flexibility of the combination of linear and time-varying effects cannot properly be depicted but it possibly obscures classical nonlinear effects [18]. Looking at the effects for a given time $t$ (we used median $\left(t_{i}\right)$ )) all covariates have the same directions of the effects as in the TSS model (cf. [3]): Effects that were estimated positive in the TSS model are also estimated positive in CoxFlexBoost, negative effect estimates were again estimated negative. However, all effects are smaller in CoxFlexBoost with respect to their norm. Note that this might not hold globally as we have additional time-varying effects that modify the given effects.

\subsubsection{Comparison of Model Selection Strategies}

Comparing the results of the application of the two-stage stepwise procedure (see [3]) and CoxFlexBoost to the Großhadern data set of patients with severe sepsis, we can conclude that both approaches have advantages with regard to different aspects:

- The two-stage stepwise procedure includes only one modeling possibility from a given set of different options, whereas CoxFlexBoost typically includes a variety of different modeling possibilities for one covariate. Thus, in the boosting context, the ability to interpret the model 
and the reliability of the model choice procedure suffer. A more sensible model choice scheme is needed in CoxFlexBoost without the selection bias in favor of time-varying effects.

- At the moment CoxFlexBoost cannot include mandatory covariates. However, such extensions could be integrated in the algorithm. The two-stage stepwise procedure is easily extended in such a way, as showed in Hofner et al. [3].

- With respect to the variable selection procedure, we can conclude that both approaches have similar outcomes. In our application CoxFlexBoost tended to a sparser solution but this could be due to the starting model with mandatory covariates in the two-stage stepwise model.

- In settings with a large number of possible predictors, CoxFlexBoost is more convenient than the two-stage stepwise procedure as it runs fully automatized. Moreover, CoxFlexBoost is able to perform variable selection and model choice in data sets with $n \ll p$ and can even select more covariates $p$ than we have got observations $n$.

Altogether, we see that none of the approaches is superior to the other. Decisions have to be based on the qualities of the algorithms in the given situation. Especially in high-dimensional settings with many possible predictors, boosting with its robustness against overfitting and the built in regularization is clearly the preferred method.

\section{$5 \quad$ Summary and Outlook}

In this paper, we derived boosting methods for flexible survival models with time-varying effects. For that purpose we used the full likelihood (and not the partial likelihood) as basis. This allows the estimation of the baseline hazard in the same framework by adding linear or smooth base-learners of time. We implemented a likelihood-based boosting approach as proposed in Tutz and Binder [20] to estimate the model. Component-wise boosting, which incorporates variable selection, has been shown to lead to appropriate models in terms of complexity. CoxFlexBoost and other likelihoodbased boosting approaches maximize in each step the likelihood of one single base-learner with an offset consisting of the estimations of all previous iterations.

A major problem in flexible survival models are the many different modeling possibilities for each covariate. It is hard to decide if a covariate should enter the model as a linear term, smooth term or as time-varying effect or if the covariate is not required at all. Boosting offers the possibility to estimate the model with inherent model choice and variable selection. To incorporate the model choice procedure in component-wise boosting, we applied the effect decompositions for smooth effects (9) and for time-varying effects (10) as proposed in Kneib et al. [12]. Furthermore, we assigned one degree of freedom to the resulting centered flexible base-learners to make the modeling possibilities comparable with respect to their flexibility (cf. Sec. 3.1 and [12]). For the differentiation of linear and smooth effects, this provides good results. However, if one tries to distinguish between linear, smooth and time-varying effects at the same time, a selection bias in favor of time-varying base-learners is observed. A possible solution could be to standardize the observed survival time that enters the model as predictor variable. This will be subject to future research.

One possible alternative to the proposed model choice scheme in CoxFlexBoost could be to fit the model in a similar fashion like that proposed in the MFPT approach by Sauerbrei et al. [8]. This means, we fit a Cox-type model with time-constant but possibly smooth effects in a component-wise boosting framework. To estimate the model one could make use of CoxFlexBoost [28] or apply the mboost package with the CoxPH() family [29]. In a second step, one could try to add time-varying effects only for the subsample of selected variables from above, where the derived model is used as starting model (i.e., as offset). Thus, base-learners for time-varying effects, for example, could be restricted to covariates without smooth effects leading to a model that is better interpretable and 
perhaps overcomes the instability issues that we discussed above. Including time-varying effects for smooth effects would result in modeling an interaction of two functions: The function of the covariate and the function of time. This can be hardly ever estimated as we typically do not have enough data to fit the resulting interaction surface.

Another issue that arises frequently in medical applications is that some covariates are of clinically high importance and thus, should be included in the model by all means. These mandatory covariates can be incorporated in the boosting framework in such a way that these variables are updated in every boosting iteration [34]. This approach could also be included in CoxFlexBoost in future work.

\section{Acknowledgments}

The authors thank W. H. Hartl from the Department of Surgery, Klinikum Großhadern for the data set and stimulating problems and D. Inthorn and H. Schneeberger for initiation and maintenance of the database of the surgical intensive care unit. B. Hofner and T. Hothorn were supported by Deutsche Forschungsgemeinschaft, grant HO 3242/1-3.

\section{References}

[1] Cox DR. Regression models and life tables (with discussion). Journal of the Royal Statistical Society. Series B 1972; 34:187-220.

[2] Moubarak P, Zilker S, Wolf H, Hofner B, Kneib T, Küchenhoff H, Jauch, K-W, Hartl WH. Activity-guided antithrombin III therapy in severe surgical sepsis: Efficacy and safety according to a retrospective data analysis. Shock 2008; 30(6):634-641.

[3] Hofner B, Kneib T, Hartl W, Küchenhoff H. Model choice in Cox-type additive hazard regression models with time-varying effects. Technical Report, Department of Statistics, Ludwig-MaximilansUniversität München 2008. URL http://epub.ub.uni-muenchen.de/3232/.

[4] Zucker DM, Karr AF. Non-parametric survival analysis with time-dependent covariate effects: A penalized likelihood approach. Annals of Statistics 1990; 18:329-352.

[5] Fahrmeir L, Kneib T, Lang S. Penalized structured additive regression: A Bayesian perspective. Statistica Sinica 2004; 14:731-761.

[6] Hastie T, Tibshirani R. Varying-coefficient models. Journal of the Royal Statistical Society. Series $B$ 1993; 55:757-796.

[7] Gray RJ. Flexible methods for analyzing survival data using splines, with application to breast cancer prognosis. Journal of the American Statistical Association 1992; 87:942-951.

[8] Sauerbrei W, Royston P, Look M. A new proposal for multivariable modelling of time-varying effects in survival data based on fractional polynomial time-transformation. Biometrical Journal 2007; 49:453-473.

[9] Abrahamowicz M, MacKenzie TA. Joint estimation of time-dependent and non-linear effects of continuous covariates on survival. Statistics in Medicine 2007; 26:392-408.

[10] Bühlmann P, Yu B. Boosting with the $L_{2}$ Loss: Regression and classification. Journal of the American Statistical Association 2003; 98:324-339.

[11] Bühlmann P, Hothorn T. Boosting algorithms: Regularization, prediction and model fitting. Statistical Science 2007; 22:477-505. 
[12] Kneib T, Hothorn T, Tutz G. Variable selection and model choice in geoadditive regression models. Biometrics 2008; (accepted).

[13] Royston P, Altman DG. Regression using fractional polynomials of continuous covariates: Parsimonious parametric modelling. Applied Statistics 1994; 43:429-453.

[14] Berger U, Schäfer J, Ulm K. Dynamic Cox modelling based on fractional polynomials: Timevariations in gastric cancer prognosis. Statistics in Medicine 2003; 22:1163-1180.

[15] Perperoglou A, le Cessie S, van Houwelingen HC. Reduced-rank hazard regression for modelling non-proportional hazards. Statistics in Medicine 2006; 25:2831-2845.

[16] Eilers PHC, Marx BD. Flexible smoothing with B-splines and penalties. Statistical Science 1996; 11:89-121.

[17] de Boor C. A Practical Guide to Splines. Springer, New York, 1978.

[18] Therneau TM, Grambsch PM. Modeling survival data: Extending the Cox model. Springer, New York, 2000.

[19] Schmid M, Hothorn T. Boosting additive models using component-wise P-splines. Computational Statistics \& Data Analysis 2008; 53:298-311.

[20] Tutz G, Binder H. Generalized additive modelling with implicit variable selection by likelihoodbased boosting. Biometrics 2006; 62:961-971.

[21] Friedman JH. Greedy function approximation: A gradient boosting machine. The Annals of Statistics 2001; 29:1189-1232.

[22] Hurvich C, Simonoff J, Tsai C. Smoothing parameter selection in nonparametric regression using an improved Akaike information criterion. Journal of the Royal Statistical Society, Series B 1998; 60:271-293.

[23] Hansen M, Yu B. Model selection and minimum description length principle. Journal of the American Statistical Association 2001; 96:746-774.

[24] Hastie T. Comment: Boosting algorithms: Regularization, prediction and model fitting. Statistical Science 2007; 22:513-515.

[25] Press WH, Teukolsky SA, Vetterling WT, Flannery B. Numerical Recipes in C: The Art of Scientific Computing. Second Edition. Cambridge University Press, 1992.

[26] Bender R, Augustin T, Blettner M. Generating survival times to simulate Cox proportional hazards models. Statistics in Medicine 2005; 24:1713-1723.

[27] R Development Core Team. R: A Language and Environment for Statistical Computing. R Foundation for Statistical Computing, Vienna, Austria 2008. URL http://www.R-project.org, ISBN 3-900051-07-0.

[28] Hofner B. CoxFlexBoost: Boosting Flexible Cox Models (with Time-Varying Effects) 2008. URL http://R-forge.R-project.org/projects/coxflexboost, R package version 0.5-0.

[29] Hothorn T, Bühlmann P, Kneib T, Schmid M, Hofner B. mboost: Model-Based Boosting 2008. URL http://cran.R-project.org/web/packages/mboost, R package version 1.0-4.

[30] Hartl WH, Wolf H, Schneider CP, Küchenhoff H, Jauch KW. Secular trends in mortality associated with new therapeutic strategies in surgical critical illness. Am J Surg 2007; 194:535-541. 
[31] Rüttinger D, Wolf H, Küchenhoff H, Jauch KW, Hartl WH. Red cell transfusion: an essential factor for patient prognosis in surgical critical illness? Shock 2007; 28:165-171.

[32] Müller MH, Moubarak P, Wolf H, Küchenhoff H, Jauch KW, Hartl WH. Independent determinants of early death in critically ill surgical patients. Shock 2008; 30:11-16.

[33] Brezger A, Kneib T, Lang S. BayesX: Analysing Bayesian structured additive regression models. Journal of Statistical Software 2005; 14(11):1-22. URL http://www.jstatsoft.org/v14/i11.

[34] Binder H, Schumacher M. Allowing for mandatory covariates in boosting estimation of sparse high-dimensional survival models. BMC Bioinformatics 2008; 9:14. 